\title{
Intérêt de l'abaissement du testicule non descendu chez l'adulte. Etude à propos de 259 patients
}

\author{
Hichem JALLOULI, Hammadi FAKHFAKH, Nouri REBAI, Issam BEN ABDALLAH, \\ Mohammed TRIFA, Ali BAHLOUL, Mohamed Nabil MHIRI
}

Service d'urologie, CHU Habib Bourguiba, Sfax, Tunisie

\section{RESUME}

Nous avons mené une étude rétrospective portant sur 259 patients âgés de plus de 18 ans, présentant des testicules non descendus (TND). L'objectif de cette étude est d'évaluer les résultats de l'abaissement du TND chez l'adulte du point de vue de l'amélioration de la fertilité et de la prévention de la dégénérescence maligne.

L'âge moyen des patients était de 24 ans. La maladie a été découverte dans la majorité des cas lors d'un examen médical systématique $(76,8 \%$ des cas) ou lors d'un bilan d'infertilité $(12,7 \%)$. Le TND était unilatéral dans $80,7 \%$ des cas et bilatéral dans $19,3 \%$ des cas. Parmi 37 couples notifiés dans notre série, seuls 4 avaient déjà eu des enfants $(10,8 \%)$. Le spermogramme, pratiqué chez les 33 patients consultant pour infertilité, était altéré dans tous les cas. Tous les patients ont eu un abaissement du testicule, sauf 36 patients $(13,9 \%)$ chez qui on a été amené à sacrifier le testicule car il était atrophique (27 cas), tumoral ( 8 cas) ou nécrosé suite à une torsion (1 cas). L'étude histologique du TND a été pratiquée chez 53 patients, montrant des altérations histologiques dans tous les cas.

L'évolution tardive a été marquée par la survenue d'une atrophie testiculaire après l'orchidopexie chez 6 patients ( $2,7 \%$ des cas), par une dégénérescence maligne opérée secondairement chez 3 patients $(1,3 \%)$, et par une amélioration des paramètres spermatiques chez 16 patients, soit $48,5 \%$ des patients consultant pour infertilité. De plus, 4 patients ont eu des enfants après traitement.

A notre sens, l'abaissement du TND chez l'adulte pourrait entraîner une amélioration de la spermatogenèse et de la fertilité. II pourrait aussi s'associer à une baisse relative de l'incidence de la dégénérescence maligne. Mais surtout, il permet une surveillance clinique plus aisée à la recherche d'une éventuelle cancérisation.

Mots clés : testicule non descendu, cryptorchidie, ectopie testiculaire, orchidopexie, infertilité masculine, cancer du testicule

\section{INTRODUCTION}

Le testicule non descendu (TND) est une anomalie congénitale fréquente, le plus souvent diagnostiquée et traitée pendant l'enfance. Cependant, à cause de l'ignorance de cette pathologie ou de la négligence de la part du patient et de ses parents, cette pathologie se voit encore à l'âge adulte. Elle pose ainsi un problème d'attitude thérapeutique : faut-t-il abaisser le testicule à cet âge assez avancé, ou bien le sacrifier?

L'objectif de cette étude est d'évaluer les résultats de l'abaissement du TND chez l'adulte du point de vue de l'amélioration de la fertilité et de la prévention de la dégénérescence maligne.

\section{MATÉRIEL ET MÉTHODES}

II s'agit d'une étude rétrospective menée sur une période de 23 ans (de 1983 à 2005). Elle concerne 259 patients dont l'âge est supérieur à 18 ans et présentant un ou deux testicules non descendus.

\section{RESULTATS}

L'âge moyen de nos patients était de 24 ans, avec des extrêmes allant de 18 à 63 ans. Par ailleurs, on a noté 3 pics de fréquence à 18,20 et à 25 ans (Figure 1).

Dans la majorité des cas, le diagnostic a été porté lors d'un examen médical systématique (199 patients soit $76,8 \%$ ) comme l'examen médical scolaire (42 patients),

\footnotetext{
Correspondance :

Dr Hichem JALLOULI - Service d'Urologie, CHU Habib Bourguiba, rue Majida Boulila, 3029 Sfax, Tunisie -

Tel +21697855744 - Fax +216 74243427 -

Email : hichemjallouli@yahoo.fr
} 


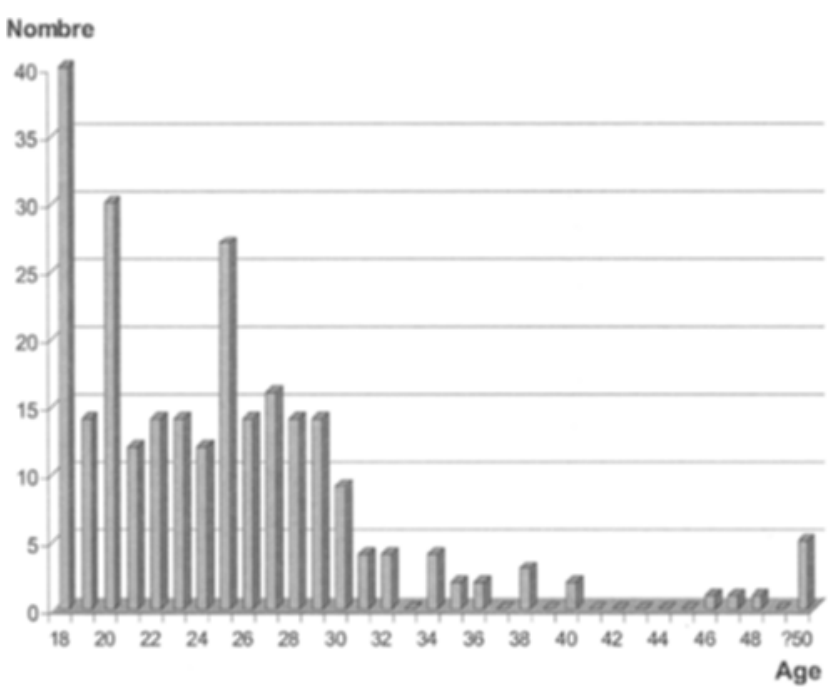

Figure 1: Répartition des patients selon l'âge. $? 50=50$ ans et plus.

l'examen de recrutement pour le service militaire (88 patients) et l'examen médical prénuptial (69 patients). Le diagnostic a aussi été porté lors d'un bilan d'infertilité chez 33 patients $(12,7 \%)$, lors d'une dégénérescence maligne du testicule chez 8 patients $(3,1 \%)$, et lors d'une torsion testiculaire chez 2 patients $(0,8 \%)$. Enfin, chez 17 patients $(6,6 \%)$, l'anomalie était connue dès la naissance mais négligée par les parents, le patient se présentant de lui-même à un âge avancé.

A l'examen clinique, le TND était unilatéral chez 209 patients $(80,7 \%)$ et bilatéral chez $50(19,3 \%)$. II était palpable chez 210 patients ( $81 \%$ ), en position inguinale superficielle chez 167 patients, en position inguinale profonde chez 43 patients, et chez un patient le testicule était ectopique en position périnéale. Par ailleurs, chez 49 patients (19\%), le TND était unilatéral et non palpable.

Parmi 37 couples notifiés dans notre série, seuls 4 avaient pu avoir une ou plusieurs grossesses spontanées, soit un taux de paternité de 10,8\%. En outre, 33 couples avaient consulté pour infertilité primaire, d'une durée supérieure à 18 mois. Ces couples n'avaient pas d'autres causes identifiables d'infertilité associée, aussi bien chez l'homme que chez la femme. L'étude du spermogramme a objectivé des anomalies dans tous les cas, à type d'azoospermie chez 10 patients $(30,3 \%)$ dont 8 avaient une cryptorchidie bilatérale et 2 avaient une cryptorchidie unilatérale, et d'oligoasthéno-tératozoospermie sévère chez 23 patients $(69,7 \%)$ dont 4 avaient une cryptorchidie bilatérale et 19 avaient une cryptorchidie unilatérale.

Sur le plan thérapeutique, les patients présentant un TND palpable ont eu un abaissement du testicule au niveau du scrotum en un seul temps opératoire. Cependant, on a été amené à enlever le testicule chez 11 patients car il était tumoral (8 cas), nécrosé suite à une torsion (1 cas) ou atrophique (2 cas). Concernant les patients ayant un testicule non palpable, le testicule a été authentifié en intra abdominal lors d'une laparotomie exploratrice. Celui-ci a été abaissé au niveau du scrotum chez 24 patients, et sacrifié chez 25 patients car il était hypotrophique et haut situé avec un pédicule court. L'abaissement du testicule intra abdominal a été réalisé à ciel ouvert chez tous les patients selon les techniques opératoires suivantes :

1) Abaissement en un seul temps opératoire sans ligature du pédicule spermatique chez 7 patients ;

2) Abaissement en deux temps opératoires sans ligature du pédicule spermatique chez 13 patients ;

3) Abaissement selon la technique de Fowler-Stephens en un seul temps opératoire chez 4 patients.

L'examen histologique des pièces d'orchidectomie dont nous avons pu disposer (35 pièces), ainsi que ceux des biopsies testiculaires réalisées sur des testicules hypotrophiques que l'on a abaissés (18 cas), a montré les faits suivants :

a) une nette réduction de la taille des tubes séminifères, observée dans tous les cas (Figure 2) ;

b) des anomalies de la lignée germinale à type d'arrêt de la spermatogenèse à des stades différents de son évolution, et parfois l'absence totale des cellules germinales. Ces anomalies ont été notées également dans tous les cas (Figure 3 et $\mathbf{4}$ ) ;

c) des altérations des cellules Sertoli (Figure 5). Ces cellules sont hyperplasiques dans $20 \%$ des cas et atrophiques dans $4 \%$ des cas. L'atrophie des cellules de Sertoli a été notée chez des patients âgés de plus de 36 ans ou en cas de testicules intra abdominaux;

d) une hyalinose de la membrane basale et fibrose du tissu conjonctif péri tubulaire. Ces altérations ont été notées dans $67 \%$ des cas (Figure 6) ;

e) une atrophie des cellules de Leydig, observée dans $40 \%$ des cas ;

f) plus rarement, une dégénérescence maligne à type de séminome ( 5 cas) (Figure 7) et de carcinome embryonnaire (3 cas) (Figure 8).

Par ailleurs, chez trois patients ayant une cryptorchidie unilatérale, on a pratiqué une biopsie du testicule controlatéral en position normale. L'examen histologique a été normal dans deux cas. Dans le troisième cas, on a observé des lésions d'altération de la spermatogenèse avec arrêt au stade de spermatide, fibrose interstitielle 


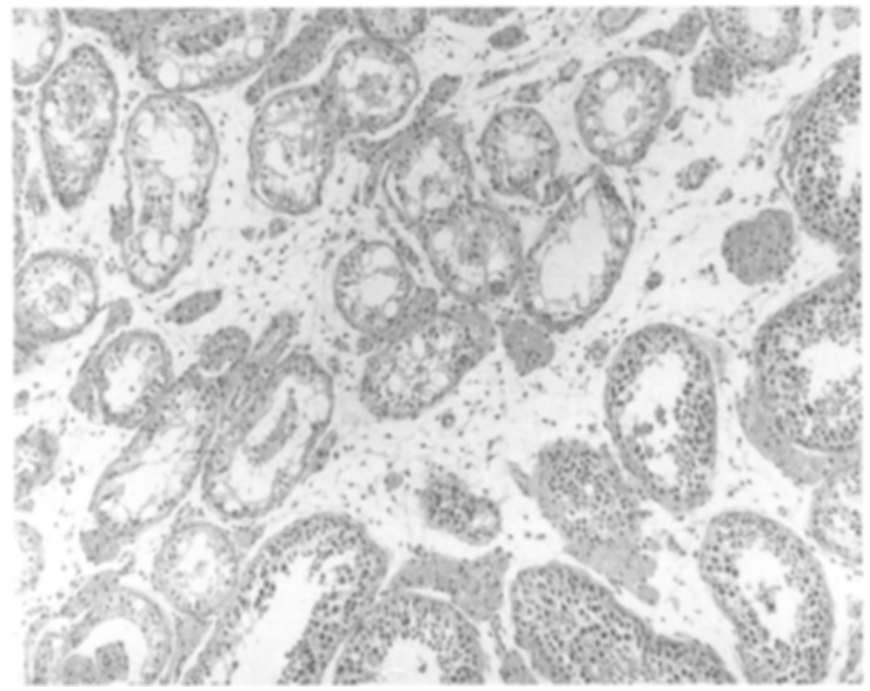

Figure 2 : Réduction de la taille des tubes séminifères.

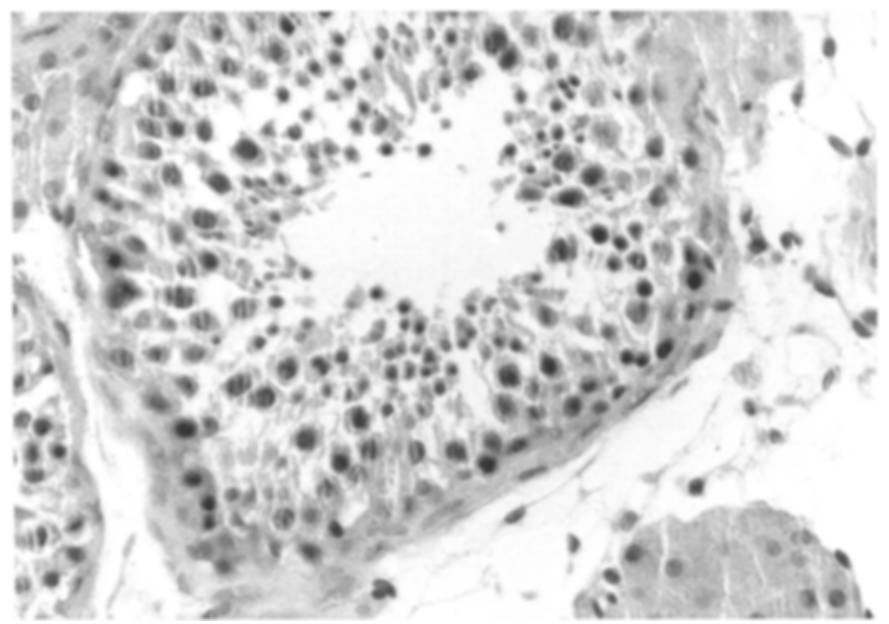

Figure 4 : Blocage de la spermatogenèse au stade spermatide.

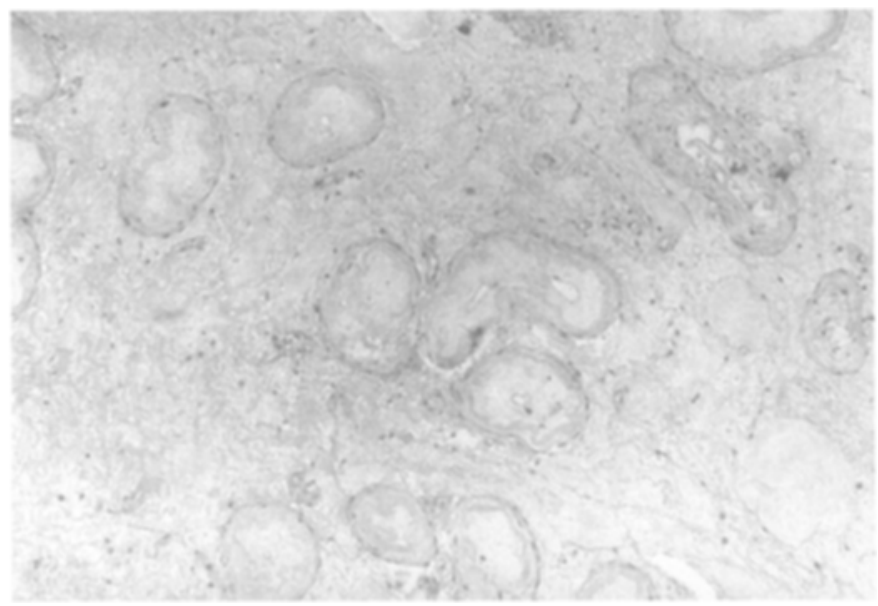

Figure 6 : Atrophie testiculaire avec hyalinose tubulaire.

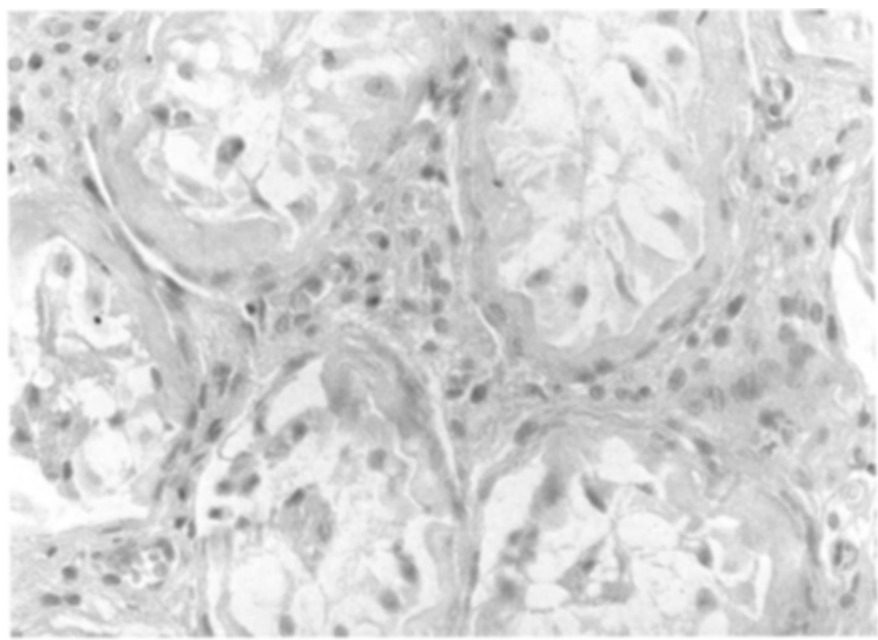

Figure 3 : Atrophie testiculaire.

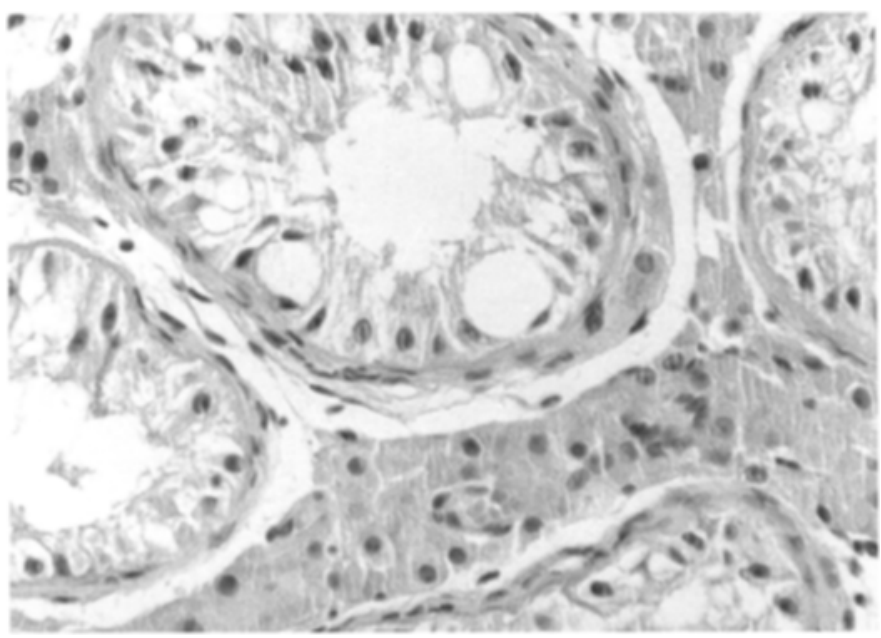

Figure 5 : Atrophie testiculaire avec hyperplasie des cellules de Leydig.

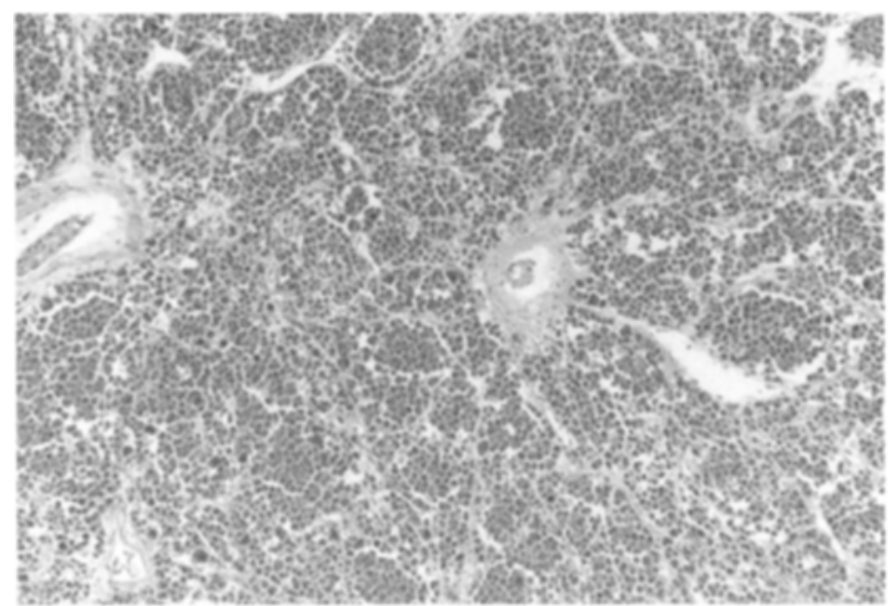

Figure 7 : Séminome. 


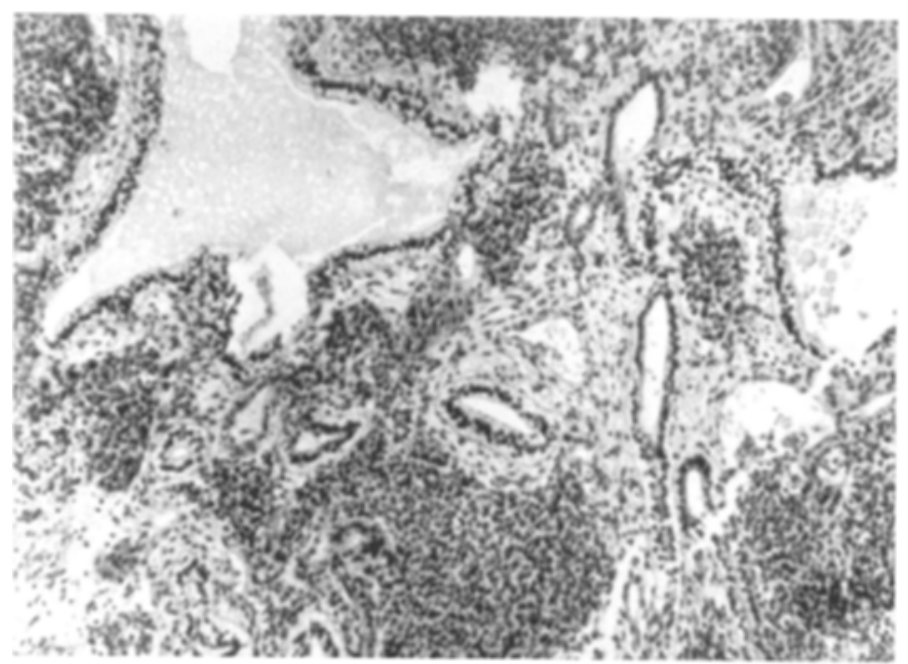

Figure 8 : Carcinome embryonaire.

péri-tubulaire, hyalinose tubulaire, et hyperplasie des cellules de Leydig.

\section{Sur le plan évolutif, on a observé :}

1) Une atrophie testiculaire chez 6 patients $(2,7 \%)$, survenue après un délai moyen de 3 ans. Elle a été observée chez des patients qui avaient un testicule intra abdominal, dont 3 ont été traités selon la technique de Fowler-Stephens en un seul temps opératoire, et 3 ayant eu un abaissement en deux temps opératoires sans ligature du pédicule spermatique.

2) Une dégénérescence maligne chez 3 patients (1,3\%), survenue après un délai moyen de 13 ans. Le type histologique était un carcinome embryonnaire intra testiculaire dans un cas, une tumeur germinale mixte dans un cas (carcinome embryonnaire et choriocarcinome), et une tumeur séminomateuse dans le troisième cas.

3) Une amélioration de la fertilité sur les données des spermogrammes post opératoires chez 16 patients, soit $48,5 \%$ des 33 patients consultants pour infertilité. Parmi ces 16 patients, onze avaient initialement un TND unilatéral $(68,8 \%)$, et cinq avaient initialement un TND bilatéral $(31,2 \%)$. De plus, quatre patients sur les 33 ont pu obtenir des enfants après traitement, soit un taux de paternité de $12,1 \%$. Ces derniers patients avaient initialement une cryptorchidie unilatérale.

\section{DISCUSSION}

Le testicule non descendu est une anomalie congénitale fréquente, sa prévalence à la naissance est estimée entre 3,7 et $4,9 \%$ des enfants à terme ; elle serait plus fréquente en cas de grossesse gémellaire et chez les prématurés $(17,2 \%$ des cas), arrivant jusqu'à $45,5 \% \mathrm{si}$ le poids à naissance est inférieur à $2 \mathrm{Kg}$. Au cours de la première année de la vie, $70 \%$ des testicules cryptorchides peuvent rejoindre leur position intra scrotale. Ainsi, la prévalence d'un TND serait de $1 \%$ après l'âge de un an $[2,11]$. Passé ce stade, cette anomalie est dans la majorité des cas traitée à un âge précoce.

Le diagnostic d'un testicule non descendu est facile, et souvent fait par les parents devant la constatation de la vacuité d'une ou des deux bourses. Cependant, par l'ignorance de cette pathologie ou par la négligence des parents, cette affection continue à se voir après l'âge de la puberté. Dans ce cas, le diagnostic est souvent fait lors des examens médicaux systématiques réalisés à titre préventif, comme l'examen médical scolaire, militaire ou prénuptial. La présence du testicule en dehors de la bourse l'expose à des altérations histologiques, et par suite à des troubles de la spermatogenèse et à un risque de dégénérescence maligne [3, $7,9,10,12,16,21]$.

Dans notre série, nous avons noté des altérations histologiques dans toutes les pièces d'orchidectomie ainsi que dans les biopsies testiculaires faites sur des testicules non descendus. Les tubes séminifères et les cellules germinales sont les structures les plus endommagées au cours de cryptorchidie $(100 \%$ des cas), suivies par les cellules de Leydig ( $40 \%$ des cas). Par contre, les cellules de Sertoli sont rarement atteintes, elles sont plutôt hyperplasiques chez l'adulte jeune. Nos résultats sont ainsi concordants avec ceux rapportés dans la littérature $[2,6,8,11,20]$.

En effet, l'altération de la lignée germinale semble apparaître dés l'âge de 6 mois et s'aggrave au cours de la deuxième année. A l'âge de 3 ans, un tiers des TND comporte un nombre normal de cellules germinales, un tiers a un nombre diminué, et un tiers un nombre sévèrement diminué. Les cellules de Leydig seraient aussi précocement atteintes dés l'âge de 3 mois. Le tissu interstitiel et les cellules de Sertoli ne seraient altérés que tardivement après l'âge de la puberté [8]. L'intensité de ces lésions est d'autant plus importante que le TND est ancien et le testicule est haut situé [13]. D'où l'intérêt d'abaisser ce testicule le plutôt possible dés sa découverte. Et si l'on tient compte de la possibilité de migration spontanée des testicules cryptorchides au cours de la première année de vie, l'âge idéal du traitement semble être donc au cours de la deuxième année.

L'examen d'un TND en microscopie électronique montre des lésions à type de transformation des mitochondries, 
de perte des ribosomes du cytoplasme et du réticulum endoplasmique, et d'augmentation des fibres de collagène dans les cellules de Sertoli et les spermatogonies [8].

De plus, l'étude du testicule intra scrotal en cas de TND unilatéral montre aussi des lésions à type de diminution du nombre des cellules germinales, un défaut de maturation et une réduction des cellules de Leydig. Ces lésions apparaissent encore dès la première année de la vie. Deux facteurs seraient impliqués dans l'atteinte du testicule controlatéral $[5,6,8]$ :

a) un phénomène endocrinien : l'altération des cellules de Leydig pourrait perturber l'équilibre hormonal entre le testicule et l'axe hypothalamo-hypophysaire, qui serait à l'origine des lésions bilatérales ;

b) un phénomène auto-immun : l'administration de drogues immunosuppressives à des souris chez les quelles on a induit une ectopie unilatérale, a permit d'éviter l'apparition des lésions controlatérales.

L'infertilité demeure une complication majeure de la cryptorchidie $[3,15,18]$. Dans la littérature, le taux d'infécondité chez les hommes ayant un TND varie de 10 à $30 \%$ dans la forme unilatérale, et de 30 à $80 \%$ pour les formes bilatérales [18].

L'étude des spermogrammes chez des patients ayant un TND unilatéral montre une azoospermie dans $14 \%$ des cas et une oligospermie dans $31 \%$ des cas. En cas de TND bilatéral, $42 \%$ des patients présentent une azoospermie et $31 \%$ présentent une oligospermie. Le taux de paternité varie de $64 \%$ à $69 \%$ dans les formes unilatérales, et de $10 \%$ à $31 \%$ dans les formes bilatérales [3]. Par contre, certains auteurs ne trouvent pas de diminution statistiquement significative de la fertilité en cas de TND unilatéral, bien que le spermogramme soit souvent altéré $[14,20]$.

La plupart des études semble montrer une amélioration du spermogramme et de la fertilité après orchidopexie en cas de TND bilatéral, même en cas d'abaissement tardif. Cependant, en cas de TND unilatéral, cette amélioration de la fertilité après orchidopexie reste encore sujette à discussion ; pour certains auteurs, la fertilité ne serait améliorée que par une intervention faite avant l'âge de deux ans. Passé cet âge, elle est peu bénéfique $[1,4]$. Dans notre série, le traitement du TND unilatéral a entraîné une amélioration du spermogramme dans $48,5 \%$ des cas et la survenue d'une grossesse dans $12,1 \%$ des cas. A notre sens, il parait donc utile de traiter la cryptorchidie même à un âge avancé pour donner au couple le maximum de chance d'avoir des enfants.

La cryptorchidie est considérée comme le facteur de risque principal des cancers testiculaires [7, 12]. Sachant que dans la population générale, le risque de développer un cancer de testicule est de 0,3 à $0,7 \%$, le risque de transformation maligne d'un TND est estimé à 3 à $5 \%$, soit 10 à 14 fois le risque d'un testicule en position normale [21]. Par ailleurs, il semble que plus le testicule est en position haute, plus le risque de cancérisation est grand. Ainsi, il serait 4 fois plus important si le testicule est intra abdominal que lorsqu'il est en position inguinale basse. L'examen histologique des biopsies testiculaires faites chez des patients ayant un TND montre que 2 à $3 \%$ de ces patients ont un néoplasie intra tubulaire $[10$, $12,16]$.

Plusieurs études montrent que l'orchidopexie ne semble pas diminuer le risque de dégénérescence maligne des testicules non descendus, et que sont intérêt se limite à la surveillance et au diagnostic précoce des tumeurs testiculaires développées ultérieurement $[16,17,19]$. Dans notre série, le taux de dégénérescence des testicules cryptorchides abaissés est de 1,3\%, donc inférieur au risque de dégénérescence d'un testicule cryptorchide laissé en place.

\section{CONCLUSION}

L'abaissement du testicule non descendu chez l'adulte pourrait entraîner une amélioration de la spermatogenèse et de la fertilité. II pourrait aussi s'associer à une baisse de l'incidence de la dégénérescence maligne, et il permet une surveillance clinique plus aisée à la recherche d'une éventuelle cancérisation ultérieure.

Cependant, le meilleur traitement reste préventif, par un diagnostic et un abaissement testiculaire à un âge précoce, idéalement au cours de la deuxième année de la vie, afin de prévenir les deux complications majeures que sont la cancérisation et l'infertilité.

\section{REFERENCES}

1. BECA D.T., HADZISELIMOVIC F. : Buserelin treatment of cryptorchidism : a randomised double-blind, placebo-controlled study. J. Urol., 1992, $148:$ 617-621.

2. BERKOWITZ G., LAPIBSKI R., DOLGIN S., GAZELLA J., BODIAN C., HOLZMAN I. : Prevalence and natural history of cryptorchidism. Paediatrics, 1993, $92: 44-49$.

3. CAROPPO E., NIEDERBERGER C., ELHAHANBLY S. : Effect of cryptorchidism and retractile testes on male factor infertility : a multicenter retrospective chart review. Fertil. Steril., 2005, 83 : 1581-1584.

4. CORTES D., THORUP J.M., LINDENBERG S. : Fertility potential after unilateral orchipexy : simultaneous testicular biopsy and orchiopexy in a cohort of 87 patients. J. Urol., 1996, $155: 1061-1065$.

5. FONTAINE E., HAJRI M., BEURTON D. : Cryptorchidie - 
Editions techniques - Encyc. Méd. Chir. (Paris-France), Néphrologie-Urologie, 18-620-A-10, 1995.

6. GERAUD M., GRAPPING C., AUDRY G. : Etude anatomique des ectopies bilatérales et du testicule controlatéral à une ectopie unilatérale. Chir. Pédiatr., 1989, 30 : 140-141.

7. GIWERCMAN A., GRINSTED J., HANSEN B., JENSEN O., SHAKKABAEK N. : Testicular cancer risk in boys with maldescended testis, a cohort study. J. Urol., 1987, 138 : 1214-1216.

8. GRACIA J., GONZALEZ N., GOMEZ M.E., PLAZA L., SANCHEZ J., ALBA J. : Clinical and anatomical study of 2000 cryptorchid testis. J. Urol., 1995, $75: 697-701$.

9. HADDAD O., LEROY X., LEMAITRE L., BISERTE J., RIGOT J.M. : Infertilité et tumeur du testicule : à propos de 25 patients. Prog. Urol., 2005, $15: 1096-1100$.

10. HAILLOT O., FETISSOF F., JANIN P., LANSON Y. : Carcinome in situ du testicule. Progr. Urol., 1992, 2 : 680688.

11. HUFF D.S., HADZISELIMOVIC F., SNYDER H., BLYTH B., DUCKETT J.W. : Early postnatal testicular maldevelopment in cryptorchidism. J. Urol., 1991, $146: 624-626$.

12. HUSMANN D.A.: Cryptorchidism and its relationship to testicular neoplasia and microlithiasis. Urology, 2005, 66 : 424-426.

13. JOHN RADCLIFFE HOSPITAL CRYPTORCHIDISM STUDY GROUP : Cryptorchidism : a prospective study of 7500 consecutive male births, 1984-8. Arch. Dis. Child., 1992, 67: 892-899.

14. LEE P.A., OLEARY L.A., SONGER N.J. : Paternity after cryptorchidism : lack of correlation with age at orchidopexy. Br. J. Urol., 1995, $75:$ 704-707.

15. LEE P.A. : Fertility after cryptorchidism : epidemiology and other outcome studies. Urology, 2005, 66 : 427-431.

16. MOTTET N. : Tumeurs des organes génitaux externes : Facteurs de risque du cancer du testicule et mécanismes de la carcinogénèse. Progr. Urol., 2003, 13 : 1244-1245.

17. NJEH M., KOUBAA A., MASMOUDI M.L., HADJ KACEM F., JLIDI R., MHIRI M.N. : Le cancer du testicule cryptorchide. J. Urol., 1995, $101:$ 177-180.

18. OH J., LANDMAN A., ALMEX E., YAN Y. : Management of the post pubertal patient with cryptorchidism : an update analysis. J. Urol., 2002, $167:$ 1329-1333.

19. PIKE M.C., CHILVERS C., PECKHAM M.J. : Effect of age at orchiodopexy on risk of testicular cancer. Lancet, 1986 1246-1248.

20. RUSNACK I., HIS-YANG W.U., DALE S.H., HOWARD M. : Histopathology in boys with cryptorchism correlates with future fertility potential. J. Urol., 2003, $169: 659-662$.

21. SWERDLOW A.J., HIGGINS C.D., PIKE M.C. : Risk of testicular cancer in a cohort of boys with cryptorchidism. Br. Med. J., 1997, $314:$ 1507-1511.

Manuscrit reçu : septembre 2006 ; accepté mai 2007.

\section{ABSTRACT}

Cryptorchidism : value of orchidopexy in adults

Hichem JALLOULI, Hammadi FAKHFAKH, Nouri REBAI, Issam BEN ABDALLAH, Mohammed TRIFA, Ali BAHLOUL, Mohamed Nabil MHIRI

Introduction : Undescended testis is a frequent congenital disease, more often diagnosed and treated during childhood. However, due to ignorance or negligence, this disease can be seen even after puberty, when it raises a therapeutic problem: is orchidopexy still useful?

This study was designed to evaluate the outcome of orchidopexy at adulthood in terms of improvement of fertility and prevention of malignant degeneration.

Material and methods : Retrospective study performed over a 23-year period (1983-2005). We have found 259 patients with undescended testis diagnosed and treated after the age of 18 years.

Results : The mean age of patients was 24 years (range: 18-63). In the majority of cases, undescended testis was diagnosed at a systemic medical examination in 199 patients $(77 \%$ of cases), and in a context of infertility in 33 patients, testicular malignancy in 8 patients, testicular torsion in 2 patients and, in 17 cases, undescended testis had been known since birth but was neglected by the parents.

Undescended testis was unilateral in 209 cases and bilateral in $\mathbf{5 0}$ cases. Out of $\mathbf{3 7}$ couples, only $\mathbf{4}$ gave birth to children ( $10.8 \%$ paternity rate). Sperm analysis was abnormal in all infertile patients. All patients were treated by orchidopexy, except for 36 patients in whom orchidectomy was performed due to testicular atrophy $(27$ cases), malignancy (8 cases) or necrosis (1 case). Biopsy of the intrascrotal testis was performed in 3 patients with unilateral cryptorchidism. Histological examination was normal in two cases and abnormal in one case. The longterm outcome was characterized by:

1. Testicular atrophy in 6 patients $(2.7 \%$ of cases).

2. Progression to malignancy in 3 patients ( $1.3 \%$ of cases).

3. Improvement of sperm parameters in 16 of 33 infertile patients $(48.5 \%) ; 4$ patients fathered children after treatment.

Conclusion : Orchidopexy at adulthood can lead to improvement of infertility. It can also decrease the incidence of malignancy and facilitate clinical examination looking for possible malignancy. However, the best treatment remains preventive, based on early diagnosis and orchidopexy.

Key-words: undescended testis, cryptorchidism, orchidopexy, ectopic testis, male infertility, testis neoplasia 